\title{
Graded elastic meta-waveguides for rainbow reflection, trapping and mode conversion
}

\author{
Jacopo Maria De Ponti (D), Luca Iorio (D, and Raffaele Ardito(D) \\ Politecnico di Milano, Department of Civil and Environmental Engineering, Piazza Leonardo da Vinci 32, 20133 Milano, Italy
}

Received: 16 October 2021 / Accepted: 27 January 2022

\begin{abstract}
Precise control of elastic waves is a challenge for many applications in the field of mechanical vibrations, ultrasonic inspection, and energy harvesting. Graded arrays of resonators on elastic substrates recently revealed superior performances for broadband wave trapping and mode conversion. In this study we present elastic waveguides able to govern waves at different scales exploiting rainbow reflection, trapping and mode conversion. We investigate whether these mechanisms, and the associated control, can be used for energy harvesting or signal conversion devices.
\end{abstract}

Keywords: Meta-waveguides / graded resonators / rainbow effect / energy harvesting

\section{Introduction}

In the last two decades, metamaterials are receiving increasing popularity for wave control across much of physics and engineering in the field of electromagnetism $[1,2]$, acoustics [3,4], and elasticity [5]. While the momentum initially focused on Bragg scattering [6,7] and subwavelength bandgaps generated by the resonance [4,8-10], the research has delivered new forms of control, encompassing tailored designs to obtain a variety of wave manipulation phenomena [11,12] including focusing and lensing [13-16], localization [17] and topological edge states $[18,19]$ amongst others.

A recent line of work employs graded arrays of resonators embedded in a host structure to manipulate wave propagation, by taking advantage of the interaction between the structure and the resonators. The term graded refers to the smooth variation of a particular parameter of the resonators along space, allowing to control the propagation of waves in a broadband regime. This modulation strategy promotes a wavenumber transformation that, in turn, activates a spatial decrease of the wave velocity when the wave enters inside the array. Array guided waves slow down as they transverse the array with different frequency components localising at specific spatial positions, resulting in a so-called trapped 'rainbow'. Originally discovered in electromagnetism using axially non-uniform, linearly tapered, planar waveguides with cores of negative index material [20], there has been a flurry of intensive research

\footnotetext{
* e-mail: jacopomaria.deponti@polimi.it
}

translating the rainbow effect into all flavors of classical wave propagation fields including acoustics [21-24], water waves [25] and fluid loaded elastic plates [26], amongst others.

In the setting of elasticity, particular advances have been recently reported in elastic devices made of arrays of resonant rods for deep elastic substrates $[27,28]$ to mode convert Rayleigh $(\mathrm{R})$ into Shear $(\mathrm{S})$ or Pressure $(\mathrm{P})$ waves. Motivated by the superior performances of graded metamaterials to trap and mode convert waves, we delineate some peculiar properties of graded meta-waveguides, namely rainbow reflection, trapping and mode conversion. Moreover, we show that rainbow trapping is intrinsically related to mode conversion and strongly dependent on the adiabaticity of the graded design.

\section{Rainbow reflection and rainbow trapping}

Elastic waveguides represent the simplest mechanical system able to support one-dimensional waves. The combination of this relative simplicity with their wide range of applications, makes them attractive candidates to study a variety of wave phenomena, defining a theoretical and experimental proof of concept framework.

Starting from the seminal work on the resonant metawedge that supports both conversion and broadband reflection [27], we have restricted the problem to waveguides with graded resonators [29-33]. These simple devices are based around gradually varying periodic arrays to take advantage of local bandgaps, to control wave propagation; array guided waves slow down as they 


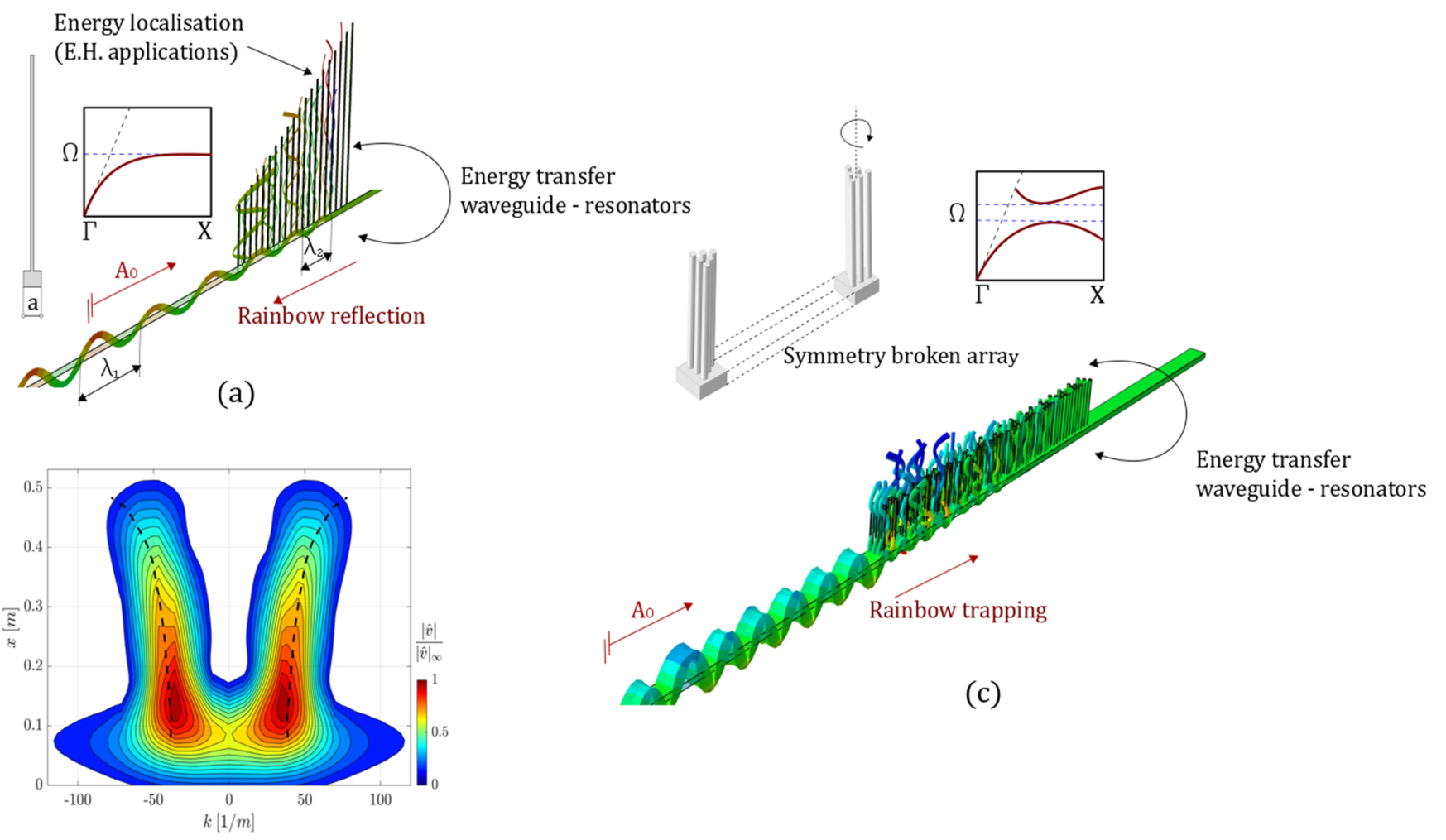

(b)

Fig. 1. (a) Elastic waveguide with a graded array of resonators of increasing height. Since the zero-group velocity mode is met at the edge of the Brillouin Zone, after a certain amount of time the input wave is reflected. While the wave propagates, the amplitude and the wavelength decrease due to energy transfer between the waveguide and the resonators. This is shown in the experimental spectrogram in (b) obtained for a rainbow reflection device in the $\mathrm{kHz}$ range. Due to this energy transfer, this mechanism is useful to harvest energy in the resonators. Contrary to rainbow reflection, rainbow trapping is obtained through a symmetry broken array, where an accidental degeneracy is lifted, resulting in zero group velocity modes within the first Brillouin zone. This allows to reduce reflections and to increase the energy available inside the array for harvesting.

transverse the array with different frequency components localising at specific spatial positions, resulting in a so-called 'trapped rainbow'. This effect originated in electromagnetism with cores of negative index material, has subsequently been achieved in a host of wave regimes, without explicit analogue to a negative index, where a surface is structured by discrete, often subwavelength, unit cells comprising resonant elements. The underlying physics, fundamental to the design of graded structures capable of spatial segregation of frequency components, for either trapping or mode conversion applications, relies on the ability to isolate the dispersion curves of the locally periodic structures which make up such an array. Depending on the position of the zero-group velocity mode in the wavenumber space, we can delineate between rainbow reflection and trapping [30], with consequently different effects concerning energy harvesting applications [29-31]. Figure 1 shows the behavior of rainbow reflection and rainbow trapping meta-waveguides. Rainbow reflection is obtained leveraging graded structures with zerogroup velocity modes at the band edge. Contrary, rainbow trapping must be located in graded structures at wavevectors within the first Brillouin Zone and hence rely on the decoupling of orthogonal eigensolutions, or symmetry breaking of the array geometry so to lift accidental degeneracies within the first Brillouin Zone. In the case of rainbow reflection, the propagating wave couples with the standing resonance mode of the resonators, creating hybrid modes with zero-group velocity regions at the band edge. When the $\mathrm{A}_{0}$ mode propagates from short to high resonators, the wave is slowed down and the wavelength is reduced, due to a progressive transfer of energy between the waveguide and the resonators. While this mechanism is useful to harvest energy inside the array, it has the drawback that after a certain amount of time waves are reflected, due to the position where the group velocity vanishes (at the end of the Brillouin Zone). The wavefield modification along the waveguide is elucidated in the experimental spectrogram of Figure 1b obtained from a rainbow reflection device in the $\mathrm{kHz}$ range.

This spectrogram is computed through a 2D Fourier Transform (FT) of the velocity field, properly windowed with a moving Gaussian function along the wave propagation direction $(x)$, which results in the function $\hat{v}_{z}(k, x, f)$. The dependence upon frequency is eliminated by taking the RMS value in time, which allows us to define the spectrogram as the amplitude $\left|\hat{v}_{z}(k, x)\right|$, displayed with 


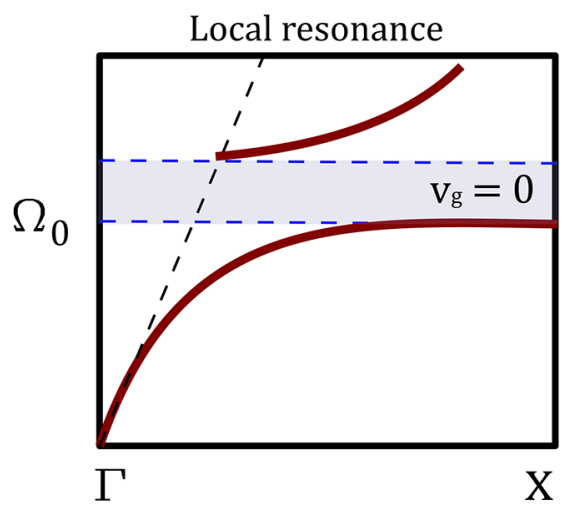

(a)

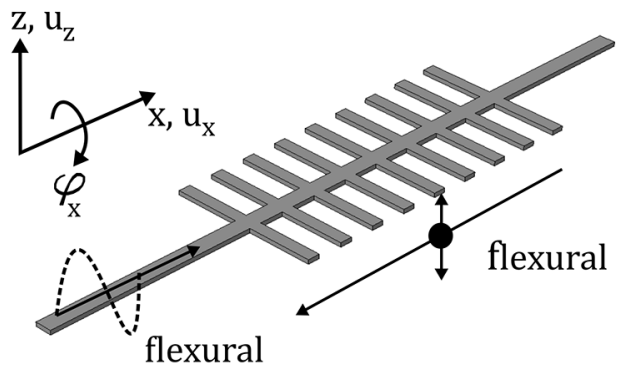

(c)

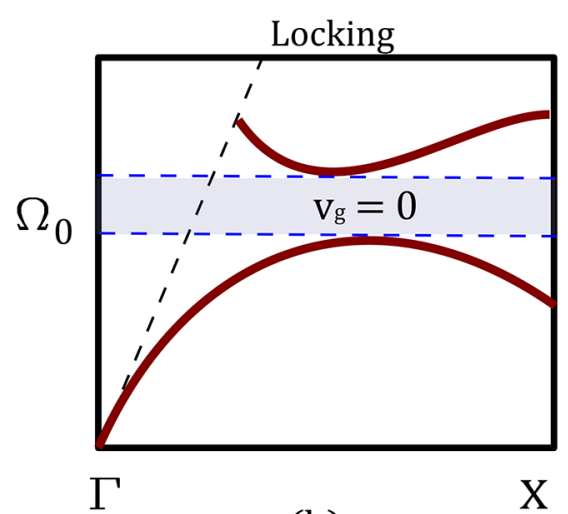

(b)

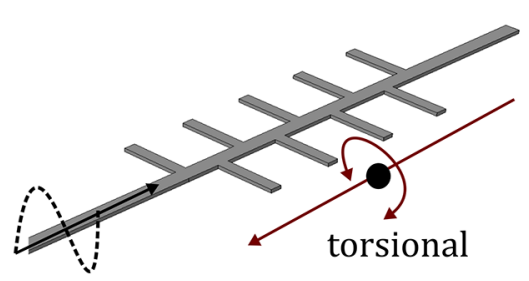

flexural

Fig. 2. (a) Dispersion relation for a periodic array of resonators placed symmetrically with respect to the wave propagation direction $(x)$. A local resonance bandgap opens at the frequency $\Omega_{0}$ due to the coupling between the propagating wave on the waveguide and the resonant mode of the resonators. The bandgap opens at the edge of the Brillouin Zone, with a relatively large zero-group velocity region. (b) Dispersion relation for aperiodic array with asymmetric resonators with respect to the wave propagation direction $(x)$. In such a case, a zero-group velocity mode arise within the Brillouin Zone due to the coupling between flexural ( $z$-polarized) and torsional $\left(\phi_{z}\right.$-polarized) waves with opposite group velocity. (c,d) Arrangement of resonators corresponding to the dispersion relations in (a) and (b) respectively.

colored contours in Figure 1b, confirming that wave propagation occurs with wavenumber transformation. The associated wavelength varies along the $x$-direction of the host beam, whereby its variation is accompanied by amplitude decrease. This behavior is further confirmed by comparison between the expected wavenumber transformation (obtained with the numerical model and represented with black dashed lines), which is superimposed to the experimental spectrogram.

Figure 1c shows a symmetry broken array, where an accidental degeneracy is lifted, resulting in zero group velocity modes within the first Brillouin Zone. By combining the grading with symmetry breaking configurations, for instance rotating the resonators inside the unit cell, rainbow trapping can be achieved. Differently with respect to rainbow reflection, which is linked to the coupling of the propagating mode with a standing resonance mode, rainbow trapping is achieved exploiting mode locking [34], i.e. the coupling of distinct waves with opposite group velocity. Figure 2 delineates this difference for the case of an infinite array with equal resonators. We compare two identical waveguides, periodically loaded with a symmetric and asymmetric arrangement of resonators with respect to the wave propagation direction $(x)$. Figures $2 \mathrm{a}, 2 \mathrm{~b}$ show the dispersion relations related to the physical systems reported in Figures 2c, 2d respectively.

When the resonators are placed symmetrically, a local resonance bandgap opens due to the coupling between the propagating wave on the waveguide and the resonant mode of the resonators. On the contrary, when the same resonators are placed asymmetrically, it is possible to create a bandgap through mode locking, i.e. by coupling waves with opposite group velocity. For both cases, we assume to excite the system with a flexural wave, i.e. a wave propagating along the waveguide length $(x)$, with particle motion along $z$. In the local resonance case (Figs. 2a, 2c), flexural waves are reflected for frequencies within the bandgap. Contrary, in the case of locking (Figs. 2b, 2d) the incoming flexural waves is mode converted into a torsional wave, i.e. a wave propagating along x with particle motion $\varphi_{z}$.

While the conventional local resonance gives a zerogroup velocity mode at the edge of the Brillouin Zone, with a relatively large zero-group velocity region along the wavenumber space, in the case of locking, the zero-group velocity region is inside the Brillouin Zone and more localized along the wavenumber space. 


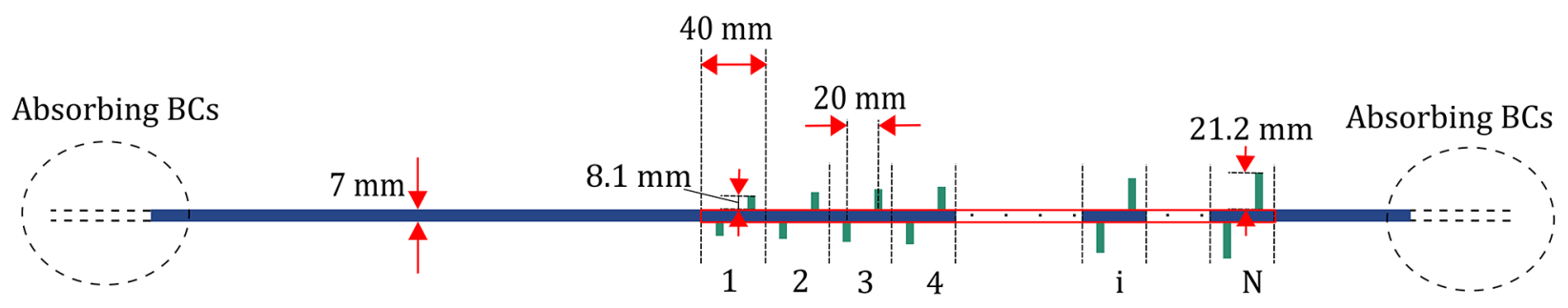

(a)

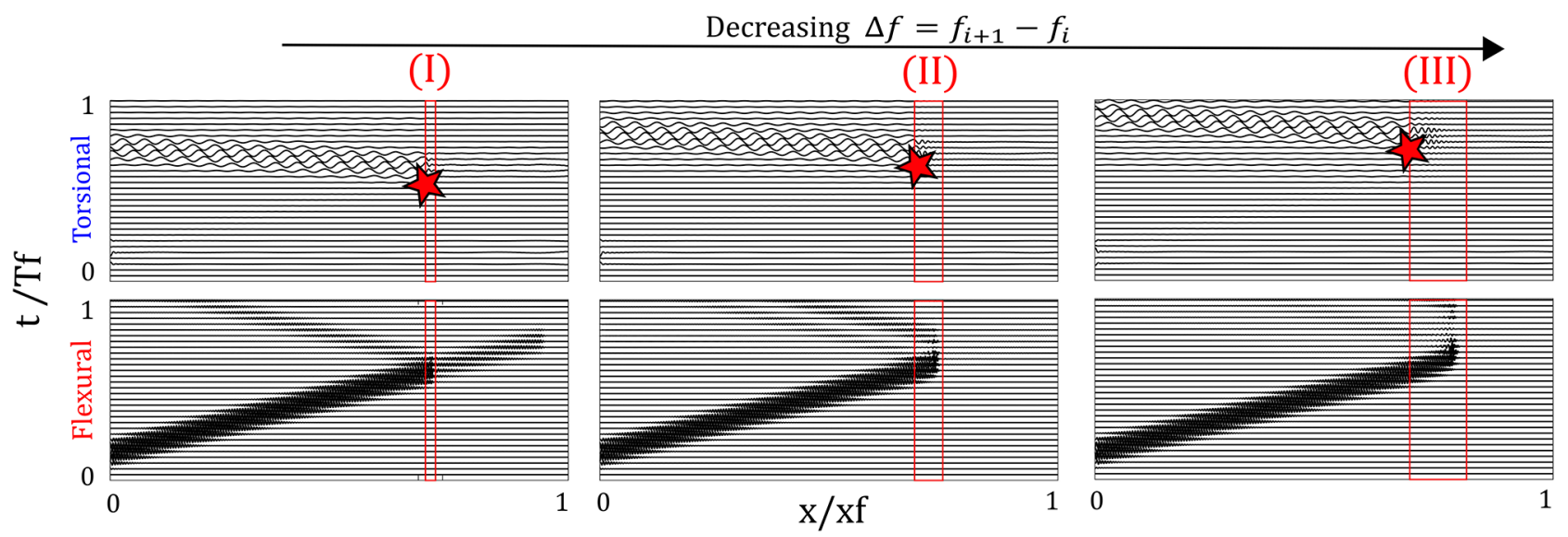

(b)

Fig. 3. (a) Graded array of asymmetric resonators with linearly increasing lengths. The spatial modification of the resonant frequency of the resonators allows to obtain locking in different spatial positions depending on frequency. (b) Space-time waterfall plots illustrating the vertical displacement (flexural) and the rotation (torsional) along the waveguide. The array of elements is highlighted with a red box. The numerical simulations are performed for a configuration with an array of (I) 9 resonators, (II) 25 resonators, and (III) 50 pairs of graded resonators. The torsional and flexural waves represented in the diagrams are normalized by the same amplitude coefficient. By increasing the number of graded resonators, i.e from left to right, it is possible to move from locking to genuine rainbow trapping.

By grading the lengths of the resonators, it is possible to create mechanical delay-lines, where waves slow down and spend more time in the vicinity of the resonators. Figure 3a shows a graded array of asymmetric resonators with linearly increasing lengths.

The spatial modification of the resonant frequency of the resonators allows to obtain locking in different spatial positions depending on frequency, i.e. rainbow trapping. In order to quantify the effect of adiabaticity of the grading for rainbow trapping, we consider three configurations with the same initial and final resonator, but different number of cells. By increasing the number of cells, the variation of the resonant frequency $\Delta f$ between adjacent resonators decreases, thus increasing the level of adiabaticity. Figure $3 \mathrm{~b}$ shows the flexural and torsional wavefield corresponding to the structure in Figure 3a. Numerical simulations are performed for a configuration with an array of (I) 9 resonators, (II) 25 resonators, and (III) 50 pairs of graded resonators. It can be noticed that trapping of the incoming flexural waves is strongly dependent on the grading. By decreasing the frequency shift between adjacent resonators, i.e. $\Delta f=f_{i+1}-f_{i}$, it is possible to move from conventional locking to genuine rainbow trapping. However, since rainbow trapping is obtained by properly coupling different modes, it is not possible to trap all the waves, but only a selective part of them. This can be noticed in Figure 3b, where for small $\Delta f$ all the incoming flexural waves is trapped, while the torsional wave is reflected with a time delay due to the group velocity decrease inside the graded structure.

\section{Conclusions}

Efficient wave manipulation is demonstrated leveraging elastic waveguides with graded arrays of resonators. Depending on the grading and the position of zero-group velocity modes in the wavenumber space, it is possible to delineate between rainbow reflection and rainbow trapping. The delineation of these two effects allows to characterise the behaviour of elastic waveguides with resonators and predict their capabilities for trapping, mode conversion and focussing of energy. 
The authors wish to thank the support of the H2020 FETproactive project MetaVEH under grant agreement No. 952039

\section{References}

1. J.B. Pendry, Negative refraction makes a perfect lens, Phys. Rev. Lett. 85, 3966 (2000)

2. J.B. Pendry, A.J. Holden, D.J. Robbinsand, W.J. Stewart, Magnetism from conductors and enhanced nonlinear phenomena, IEEE Trans. Microw. Theory Tech. 47, 2075 (1999)

3. R.V. Craster, S. Guenneau, Acoustic Metamaterials, Negative Refraction, Imaging, Lensing and Cloaking, (Springer Series in Materials Science, Berlin, 2013), pp. 1-324

4. Z. Liu, X. Zhang, Y. Mao, Y.Y. Zhu, Z. Yang, C.T. Chan, P. Sheng, Locally resonant sonic materials, Science 289, 1734 (2000)

5. R.V. Craster, S. Guenneau, World Scientific Handbook of Metamaterials and Plasmonics: Volume 2: Elastic, Acoustic and Seismic Metamaterials (World Scientific, Singapore, 2017)

6. J.O. Vasseur, P.A. Deymier, B. Chenni, B. Djafari-Rouhani, L. Dobrzynski, D. Prevost, Experimental and theoretical evidence for the existence of absolute acoustic band gaps in two-dimensional solid phononic crystals, Phys. Rev. Lett. 86, 3012 (2001)

7. V. Laude, Phononic Crystals Artificial Crystals for Sonic, Acoustic, and Elastic Waves (De Gruyter, Berlin, 2015)

8. A.E. Miroshnichenko, S. Flach, Y.S. Kivshar, Fano resonances in nanoscale structures, Rev. Mod. Phys. 82, 2257 (2010)

9. F. Lemoult, M. Fink, G. Lerosey, Acoustic resonators for farfield control of sound on a subwavelength scale, Phys. Rev. Lett. 107, 064301 (2011)

10. E.G. Williams, P. Roux, M. Rupin, W.A. Kuperman, Theory of multiresonant metamaterials for A0 Lamb waves, Phys. Rev. B 91, 104307 (2015)

11. Y. Liu, Z. Liang, F. Liu, O. Diba, A. Lamb, J. Li, Source illusion devices for flexural lamb waves using elastic metasurfaces, Phys. Rev. Lett. 119, 034301 (2017)

12. P. Packo, A.N. Norris, D. Torrent, Inverse grating problem: efficient design of anomalous flexural wave reflectors and refractors, Phys. Rev. Appl. 11, 014023 (2019)

13. X. Yan, R. Zhu, G. Huang, F.G. Yuan, Focusing guided waves using surface bonded elastic metamaterials, Appl. Phys. Lett. 103, 121901 (2013)

14. A. Colombi, Resonant metalenses for flexural waves, J. Acoust. Soc. Am. 140, EL423 (2016)

15. R. Fuentes-Domínguez, M. Yao, A. Colombi, P. Dryburgh, D. Pieris, A. Jackson-Crisp, D. Colquitt, A. Clare, R.J. Smith, M. Clark, Design of a resonant Luneburg lens for surface acoustic waves, Ultrasonics 111, 106306 (2021)

16. G.J. Chaplain, R.V. Craster, Flat lensing by graded line meta-arrays, Phys. Rev. B 99, 220102(R) (2019)

17. M. Lott, P. Roux, L. Seydoux, B. Tallon, A. Pelat, S. Skipetrov, A. Colombi, Localized modes on a metasurface through multiwave interactions, Phys. Rev. Mater. 4, 065203 (2020)
18. R.K. Pal, M. Ruzzene, Edge waves in plates with resonators: an elastic analogue of the quantum valley Hall effect, New J. Phys. 19, 025001 (2017)

19. Y. Xia, A. Erturk, M. Ruzzene, Topological edge states in quasiperiodic locally resonant metastructures, Phys. Rev. Appl. 13, 014023 (2020)

20. K.L. Tsakmakidis, A.D. Boardman, O. Hess, 'Trapped rainbow' storage of light in metamaterials, Nature $\mathbf{4 5 0}$, 397 (2007)

21. J. Zhu, Y. Chen, X. Zhu, F.J. Garcia-Vidal, X. Yin, W. Zhang, X. Zhang, Acoustic rainbow trapping, Sci. Rep. 3, 1728 (2013)

22. V. Romero-García, R. Picó, A. Cebrecos, V.J. SánchezMorcillo, K. Staliunas, Enhancement of sound in chirped sonic crystals, Appl. Phys. Lett. 102, 091906 (2013)

23. A. Cebrecos, R. Picó, V.J. Sánchez-Morcillo, K. Staliunas, V.V. Romero-García, L.M. Garcia-Raffi, Enhancement of sound by soft reflections in exponentially chirped crystals, AIP Adv. 4, 124402 (2014)

24. Y. Chen, H. Liu, M. Reilly, H. Bae, M. Yu, Enhanced acoustic sensing through wave compression and pressure amplification in anisotropic metamaterials, Nat. Commun. 5, 5247 (2014)

25. L.G. Bennetts, M.A. Peter, R.V. Craster, Graded resonator arrays for spatial frequency separation and amplification of water waves, J. Fluid Mech. 854, R4 (2018)

26. E.A. Skelton, R.V. Craster, A. Colombi, D.J. Colquitt, The multi-physics metawedge: graded arrays on fluid-loaded elastic plates and the mechanical analogues of rainbow trapping and mode conversion, New J. Phys. 20, 053017 (2018)

27. A. Colombi, D. Colquitt, P. Roux, S. Guenneau, R.V. Craster, A seismic metamaterial: the resonant metawedge, Sci. Rep. 6, 27717 (2016)

28. G.J. Chaplain, J.M. De Ponti, A. Colombi, R. FuentesDominguez, P. Dryburg, D. Pieris, R.J. Smith, A. Clare, M. Clark, R.V. Craster, Tailored elastic surface to body wave Umklapp conversion, Nat. Commun. 11, 3267 (2020)

29. J.M. De Ponti, A. Colombi, R. Ardito, F. Braghin, A. Corigliano, R.V. Craster, Graded elastic metasurface for enhanced energy harvesting, New J. Phys. 22, 013013 (2020)

30. G.J. Chaplain, D. Pajer, J.M. De Ponti, R.V. Craster, Delineating rainbow reflection and trapping with applications for energy harvesting, New J. Phys. 22, 063024 (2020)

31. J.M. De Ponti, A. Colombi, E. Riva, R. Ardito, F. Braghin, A. Corigliano, R.V. Craster, Experimental investigation of amplification, via a mechanical delay-line, in a rainbow-based metamaterial for energy harvesting, Appl. Phys. Lett. 117, $143902(2020)$

32. J.M. De Ponti, Graded Elastic Metamaterials for Energy Harvesting (Springer, Berlin, 2021)

33. J.M. De Ponti, L. Iorio, E. Riva, R. Ardito, F. Braghin, A. Corigliano, Selective mode conversion and rainbow trapping via graded elastic waveguides, Phys. Rev. Appl. 16, 034028 (2021)

34. B.R. Mace, E. Manconi, Wave motion and dispersion phenomena: Veering, locking and strong coupling effects, J. Acoust. Soc. Am. 131, 1015 (2012)

Cite this article as: Jacopo Maria De Ponti, Luca Iorio, Raffaele Ardito, Graded elastic meta-waveguides for rainbow reflection, trapping and mode conversion, EPJ Appl. Metamat. 9, 6 (2022) 